\title{
Intérêts et limites d'une approche par type de milieu pour le développement d'un indice poisson lacustre français
}

\author{
Relevance and limits of a typological approach \\ for the development of a lake fish index in France
}

\author{
L. Launois, C. Argillier \\ Cemagref, Unité Hydrobiologie, Pôle Plans d'Eau, 3275 route de Cézanne, CS 40061, \\ 13182 Aix-en-Provence, France \\ christine.argillier@cemagref.fr
}

\begin{abstract}
Résumé - Pour évaluer l'état des masses d'eau, la Directive Cadre Européenne sur l'Eau requiert l'utilisation de bioindicateurs adaptés à des systèmes homogènes d'un point de vue environnemental. Nous présentons ici une classification de plans d'eau basée sur des paramètres environnementaux naturels connus pour structurer l'ichtyofaune. Ensuite, un premier essai de sélection de métriques piscicoles, par analyse de la réponse aux pressions anthropiques s'exerçant sur les bassins versants, est décrit pour deux types de petits plans d'eau français, i.e. les lacs naturels plutôt profonds et de forme arrondie, situés à des altitudes moyennes et des retenues peu profondes, à berges découpées et situés à très faible altitude. Les résultats obtenus sont encourageants car les richesses en espèces phytophiles et tolérantes répondent bien aux pressions anthropiques. Cependant, cette approche présente des limites. En effet, certains types de milieux identifiés dans cette étude ne regroupent que peu de plans d'eau; d'autres conservent une certaine variabilité environnementale à même d'influencer l'ichtyofaune. D'autre part, le nombre de sites pas ou peu perturbés est parfois restreint, voire nul pour le groupe des retenues peu profondes, à berges découpées, de très faible altitude. Enfin, entre les différents groupes typologiques, les gammes de pressions observées, sont elles-mêmes distinctes. Ces résultats devront être validés mais la recherche d'une solution alternative à cette approche typologique, pour évaluer l'état des plans d'eau, devrait être envisagée.
\end{abstract}

Mots clés - bioindication, communauté piscicole, lac naturel, réservoir, typologie

\begin{abstract}
In order to assess the ecological status of water bodies, the Water Framework Directive requires the development of bioindicators adapted to homogenous ecosystems with respect to their environmental conditions. A lake typology based on environmental parameters known to influence fish communities is presented; a first approach to select fish based metrics by analysing their response to the anthropogenic pressures exerted on the watershed is then described for two types of small French lakes, i.e. deep natural lakes at high altitude with shoreline development factors (SLDF) close to 1, and shallow reservoirs at low altitude, showing high SLDF. Our results are encouraging, because phytophilic and
\end{abstract}


tolerant species richnesses respond well to anthropogenic pressures. However, this approach has also limits. Indeed, some of the lake types studied are composed of only a few lakes and among other types environmental variability is still important and can influence fish communities. Moreover, the number of sites that are not or less impacted by anthropogenic pressures is sometimes restricted or even null for shallow reservoirs showing high SLDF but low altitude. Finally, anthropogenic pressure gradients themselves are distinct between the different types of lakes. These results need to be validated but an alternative approach to this typology based assessment system should be envisaged for lakes.

Key words - bioindication, fish community, lake, reservoir, typology

\section{INTRODUCTION}

Les systèmes lentiques ont par nature des caractéristiques physiques, chimiques et biologiques différentes. En effet, les processus hydrologiques, la géologie du bassin versant, la position géographique des plans d'eau et la morphologie de la cuvette lacustre, sont autant de paramètres environnementaux qui affectent les processus biologiques, l'assemblage des communautés (Plafkin et al., 1989) et qui contribuent à rendre les caractéristiques physiques et chimiques d'un plan d'eau uniques. Ce caractère unique conféré par les variables environnementales aux lacs, rend difficile le développement d'outils d'évaluation transposables à de larges échelles spatiales (Schmutz et al., 2007 ; Schupp 1992). Historiquement, le recours à une approche typologique apparaît comme le moyen le plus intuitif et adéquat pour s'affranchir de cette variabilité environnementale (Drake \& Pereira, 2002; Hawkins et al., 2000; Schupp 1992). En effet, en passant par la création de groupes de lacs homogènes d'un point de vue environnemental, on espère que les processus écologiques qui s'opèrent au sein de chacun de ces groupes soient similaires, tout comme les réponses des communautés piscicoles aux pressions anthropiques. Quel que soit le type de milieu continental et côtier, c'est ce cadre théorique qui a été retenu pour le mise en place des indicateurs à utiliser en application de la Directive Cadre Européenne sur l'Eau (DCE; Communauté européenne, 2000). Ainsi, en France une typologie des plans d'eau a été définie. Elle est basée sur l'origine naturelle ou anthropique du plan d'eau, sur l'hydroécorégion (géologie, relief et climat), la morphologie de la cuvette et pour certains types sur le fonctionnement hydraulique. Douze types de lacs naturels et 18 types de plans d'eau d'origine anthropique sont décrits (Ministère de l'Écologie et du Développement Durable, 2005).

L'objectif de ce travail est d'examiner, à partir des données d'inventaire disponibles, les possibilités de développement d'un indicateur poisson adapté aux plans d'eau français, d'origine naturelle et anthropique, dans le cadre d'une approche par type de masses d'eau, conforme aux principes généraux de la DCE. Pour cela, compte tenu de la très forte variabilité environnementale résiduelle dans les types nationaux cités précédemment (exemple de la profondeur décrit dans De Bortoli \& Argillier, 2008), 
la démarche consiste à (1) établir une nouvelle typologie physique des plans d'eau sur la base de variables environnementales, connues pour influencer l'ichtyofaune, disponibles au niveau national, puis à (2) analyser la réponse aux pressions de métriques candidates, dans les types de plans d'eau où les données relatives à l'ichtyofaune sont les plus nombreuses. $\mathrm{Ce}$ travail vise à dégager les avantages et les limites de cette approche typologique au niveau national, pour la mise au point d'un bioindicateur basé sur l'ichtyofaune des plans d'eau.

\section{MATÉRIEL ET MÉTHODES}

\subsection{Réalisation de la typologie lacustre}

Le jeu de données environnementales disponible concerne 34 lacs naturels et 104 réservoirs sur un total de 71 lacs et 368 retenues sur lesquels doit s'effectuer un rapportage au niveau européen. La localisation de ces 138 plans d'eau est présentée sur la figure 1a et leur liste en annexe 1. Ces plans d'eau sont bien répartis sur l'ensemble du territoire, mais ils sont tous situés à moins de $1500 \mathrm{~m}$ d'altitude, limite au-delà de laquelle les densités et les richesses spécifiques des poissons sont très faibles (Argillier et al., 2002) et donc potentiellement limitantes pour le calcul de certaines métriques.

Ces sites ont été décrits par cinq variables environnementales " physiques ", indépendantes entre elles et non influencées par les pressions : la surface du bassin versant, l'altitude, la surface et la profondeur maximale du plan d'eau, ainsi que l'indice de développement des berges [Shoreline
Development Factor (SLDF)] (Eadie \& Keast, 1984). Ces variables ont été log transformées. En l'absence de données homogènes de températures de l'eau, celles-ci n'étant prises que ponctuellement lors des pêches effectuées à des dates variables, la température moyenne de l'air des mois de janvier et de juillet ont été calculées à partir du modèle AURHELY, qui recense ces valeurs moyennes sur la période 1961-1990 (Benichou \& Le Breton, 1987). Elles ont été utilisées pour créer deux nouvelles variables relatives à la température non corrélées, comme pour l'indice poissons rivière : $\mathrm{T}^{\circ}$ janvier $+T^{\circ}$ juillet et $T^{\circ}$ juillet $-T^{\circ}$ janvier (Oberdorff et al., 2001, 2002; Pont et al., 2006, 2007). Ces deux variables ont été utilisées telles quelles.

Pour la réalisation de groupes de systèmes homogènes du point de vue de l'environnement naturel, les plans d'eau naturels et d'origine artificielle ont été traités séparément. Les groupes de lacs qui s'apparentent le plus du point de vue environnemental ont été définis par la réalisation d'une classification non supervisée, ascendante (méthode des K-means). Les sept variables environnementales précédemment décrites et présentées dans le tableau I ont d'abord été centrées et réduites. Ensuite, une analyse en composantes principales a été réalisée sur ces variables environnementales. Les cinq premières composantes ont été sélectionnées, car elles expliquaient plus de $90 \%$ de la variabilité. Finalement, ces cinq composantes ont été analysées selon la méthode des K-means (MacQuenn, 1967). Cette méthode partitionne les données en K groupes, ou " clusters ", ne se chevauchant pas. Ce résultat 


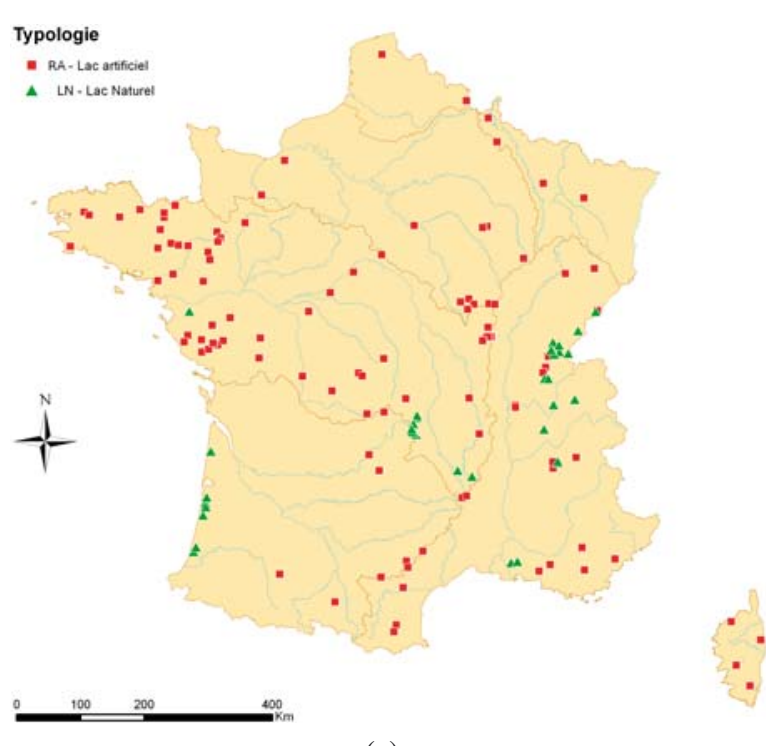

(a)

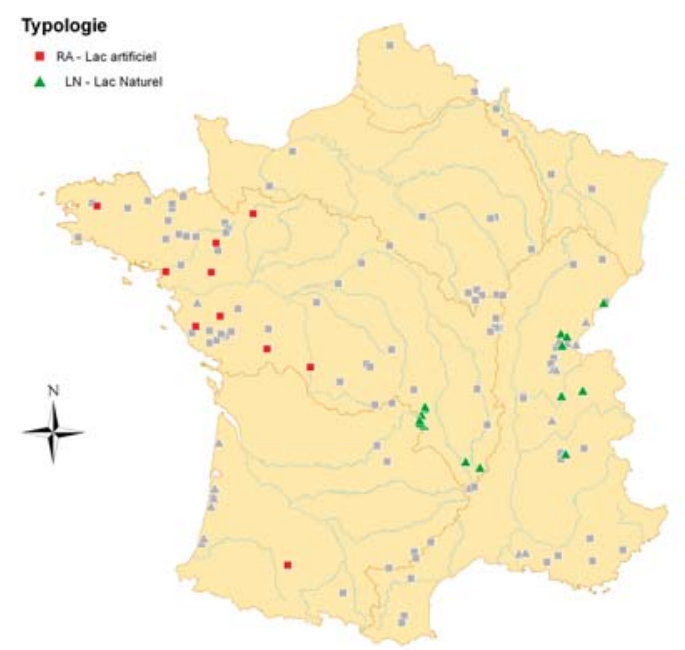

(b)

Fig. 1. Localisation géographique des plans d'eau étudiés (1a) pour la réalisation de la typologie et (1b) pour l'analyse de la réponse des métriques aux pressions. Dans ce dernier cas, les plans d'eau en grisé sont exclus.

Fig. 1. Location of the studied sites in France used for (1a) the typology approach development, and (1b) the analysis of the metric responses to anthropogenic pressures. In this case, grey tinted lakes are excluded. 


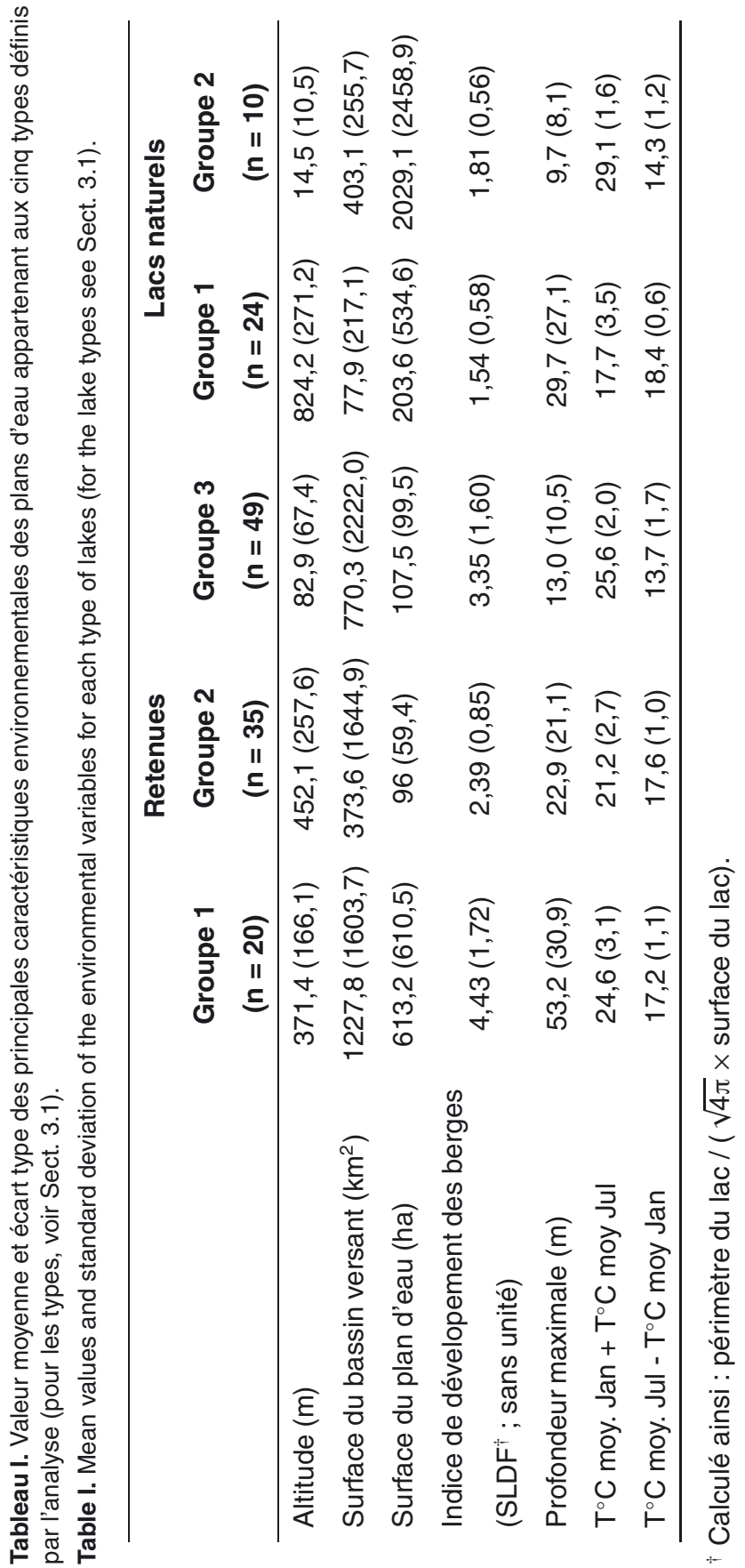




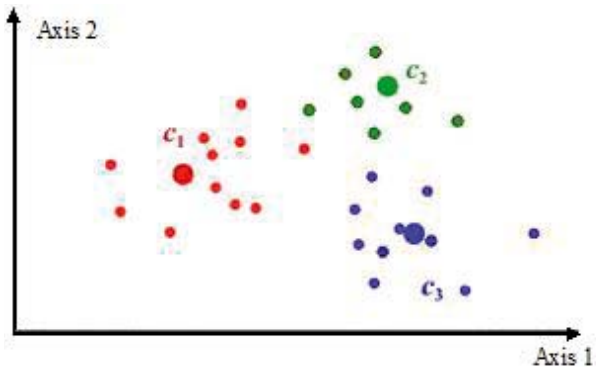

Fig. 2. Illustration de la méthode K-means, avec le partitionnement des données en 3 groupes, ou « clusters ». Ce résultat est obtenu en positionnant 3 «prototypes » (c1, c2 et c3) dans les régions de l'espace les plus peuplées. Adapté de MacQuenn (1967).

Fig. 2. Illustration of the K-means method. Data were partitioned into 3 groups or "clusters". This result is obtained after positioning 3 "prototypes" (c1, c2 and c3) in the most densely populated regions. Adapted from MacQuenn (1967).

est obtenu en positionnant $\mathrm{K}$ « prototypes » ou «centroïdes » dans les régions de l'espace les plus peuplées (Fig. 2). Chaque observation est alors affectée au prototype le plus proche (règle dite « de la distance minimale »). Chaque groupe contient donc les observations qui sont plus proches d'un certain prototype que de tout autre prototype.

\subsection{Analyse de la réponse de l'ichtyofaune aux pressions dans chaque type}

\subsubsection{Les données poissons}

L'ichtyofaune a été échantillonnée entre 2005 et 2008, sur 44 des 138 plans d'eau français sur lesquels les variables environnementales sont disponibles (voir liste en annexe 1). Le protocole utilisé est le protocole standardisé aux filets maillants
(CEN, 2005). II prévoit une campagne d'échantillonnage aléatoire stratifiée par zones de différentes profondeurs, durant la période estivale (juin à octobre, la température de l'eau en surface devant être supérieure à $15^{\circ} \mathrm{C}$ ). L'effort de pêche est défini en fonction de la profondeur et de la surface des systèmes.

Les espèces présentes ont été recensées et tous les poissons ont été comptés et pesés.

À partir de ces données, les abondances exprimées en nombre d'individus et en biomasse par unité d'effort (surface $\times$ temps de pose) ont été calculées. De plus, onze traits d'histoire de vie associés aux espèces ont été définis sur la base de données de la littérature (Balon, 1975; Bruslé \& Quignard, 2001; Keith \& Allardi, 2001), sur les bases en ligne (Pont et al., 2006) et sur avis d'experts en particulier pour les traits liés au régime alimentaire (H. Persat, N. Poulet, O. Schlumberger) (Tab. II). Ces traits ont été utilisés pour le calcul de quatorze métriques piscicoles choisies parmi celles les plus fréquemment utilisées en bioindication sur les plans d'eau et dont le calcul repose sur plus de deux espèces dans l'ensemble du jeu de données (Tab. III).

\subsubsection{Les données de pressions}

Les données de pression étaient disponibles pour 44 lacs sur lesquels l'ichtyofaune a été échantillonnée. Les pressions s'exerçant localement étant trop partielles sur l'ensemble des sites, on s'est intéressé uniquement aux pressions sur le bassin versant des plans d'eau (BV). Neuf variables de pressions (ou de forces 


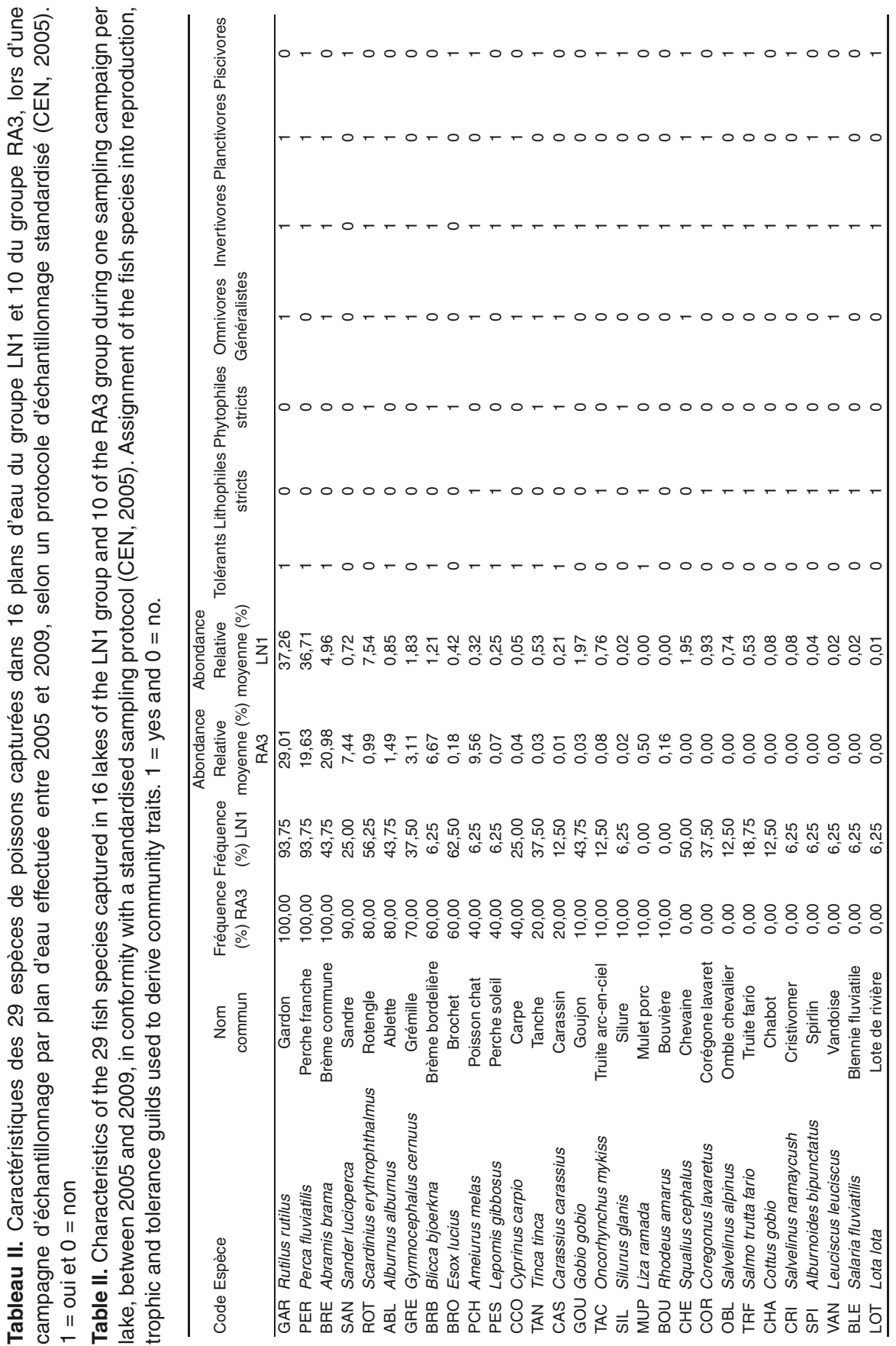




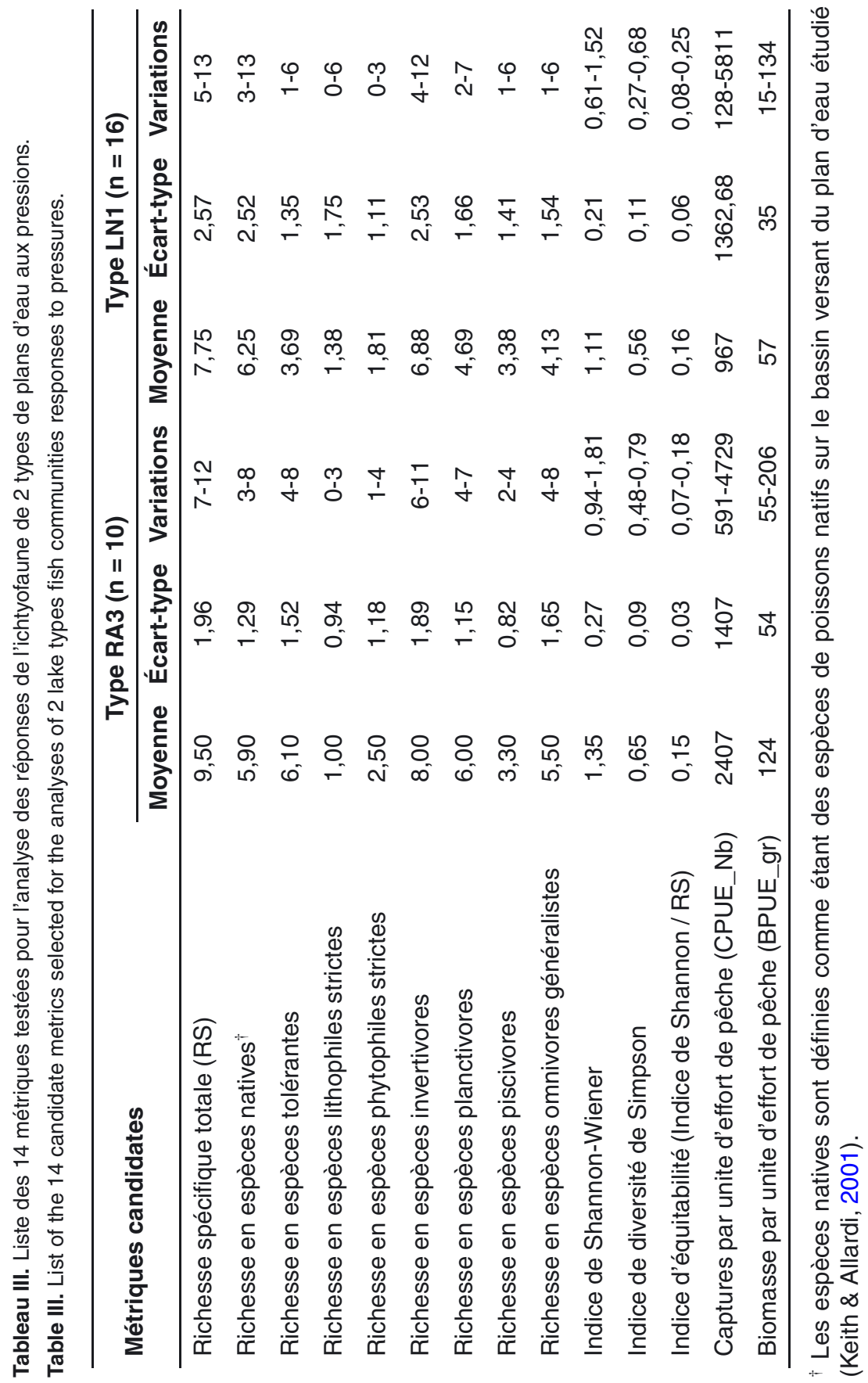


motrices à l'origine des pressions, voir IMPRESS, 2002) ont été décrites afin de construire des indices synthétiques de pressions calculables pour l'ensemble des plans d'eau. Ces variables sont :

- la surface en zones urbaines sur le $B V$, exprimée en \% de la surface totale du BV (source : Corine Land Cover 2000),

- la surface en zones agricoles sur le BV, également exprimée en \% de la surface du BV (source : Corine Land Cover 2000),

- la densité de population humaine sur le BV, en nombre d'individus à l'hectare,

- la densité de population humaine sur une zone tampon de $1 \mathrm{~km}$ autour du lac, en nombre d'individus à l'hectare,

- la densité de réseau de communication sur le BV, exprimée en $\mathrm{km} / \mathrm{km}^{2}$,

- les surplus en azote $\left(\mathrm{N}_{\text {total }}\right)$ et en phosphore $\left(P_{\text {total }}\right)$ sur le BV (source : NOPOLU - PÖYRY Environment), exprimés en tonnes/ $\mathrm{km}^{2}$,

- le bilan en azote sur le BV, exprimé en tonnes $/ \mathrm{km}^{2}$, qui correspond à la somme des surplus en azote et des dépôts atmosphériques en azote d'origine anthropique,

- l'aléa moyen d'érosion couplé aux surplus en $\mathrm{P}_{\text {total }}$ du BV (source : INRA - Ifen - GIS Sol, 2000). En effet, comme le phosphore est un élément chimique peu mobile (Carpenter et al., 1998), et comme le préconise Coale (2000), nous avons multiplié le surplus en $\mathrm{P}_{\text {total }}$ par l'aléa moyen d'érosion du BV. $\mathrm{Ce}$ dernier est un coefficient adimensionnel intégrant la battance des sols, l'érodabilité, la topogra- phie, l'occupation du sol, les précipitations, ainsi que les zones où les cultures sont prépondérantes (http://erosion.orleans.inra.fr/ alea_france_version2000/index.html).

Une analyse en composantes principales a ensuite été réalisée sur ces neuf variables. Deux nouvelles ACP ont ensuite été effectuées, l'une sur les variables liées à l'agriculture, l'autre sur les variables liées à l'urbanisme.

Les coordonnées des lacs sur l'axe principal de ces ACP constituent les indices synthétiques de pression. Ces deux indices sont ensuite sommés pour donner un indice global de pression s'exerçant sur le BV, à chaque lac. Avant d'être log transformés, ces indices sont translatés pour ne pas prendre de valeur négative. La gamme de variation des pressions a été analysée au sein de chaque type de plans d'eau.

\subsubsection{Réponse des métriques aux pressions}

Les groupes typologiques regroupant le plus de sites avec des données poissons sont le type RA3, i.e. 10 retenues peu profondes, à berges découpées et à très faible altitude et, le type LN1, i.e. 16 lacs naturels plutôt profonds et de forme arrondie, situés à des altitudes moyennes (Fig. 1b). La réponse des métriques aux pressions a été testée sur ces deux types par régression linéaire entre les indices de pressions et les métriques (coefficient de corrélation de Spearman $(\rho), P<$ $0,05)$.

Toutes les analyses ont été réalisées sous « $R$ » (Ihaka \& Gentleman, 1996; R Development Core Team, 2008). 


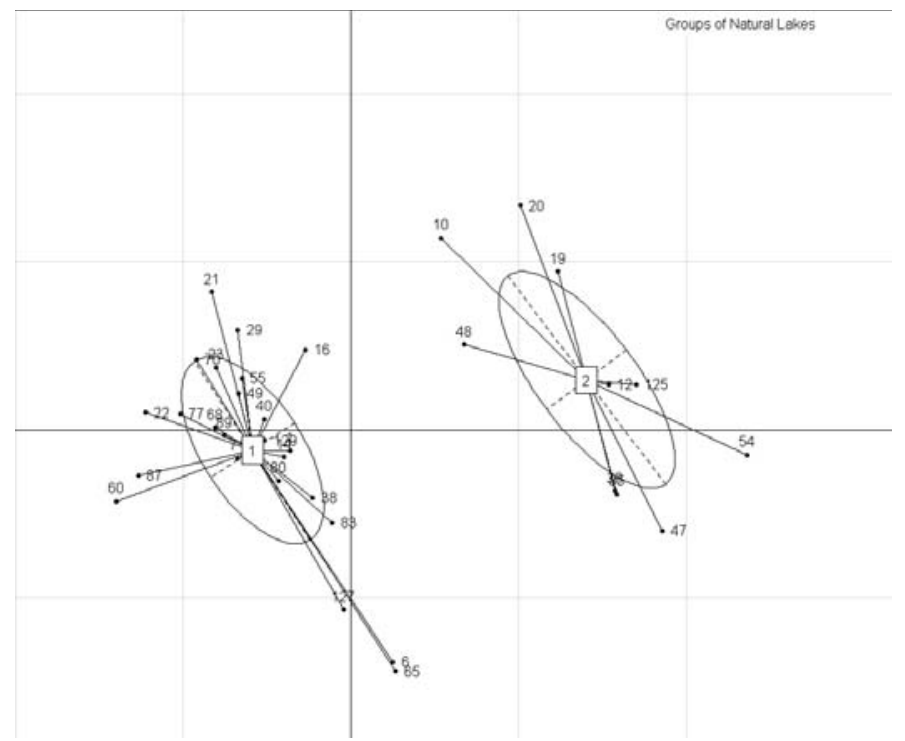

Fig. 3. Identification des deux groupes de lacs naturels (1: groupe LN1; 2 : groupe LN2) par la méthode des K-means - analyse sur 34 lacs naturels. Les axes horizontal et vertical correspondent, respectivement au premier et au deuxième axe de l'ACP réalisée sur les sept variables environnementales disponibles pour les 34 lacs naturels. Pour les numéros des plans d'eau, voir la liste détaillée à l'annexe 1.

Fig. 3. Identification of two types of natural lakes (1: LN1 group; 2: LN2 group) using the K-means approach. 34 natural lakes were analysed. Horizontal and vertical axes correspond, respectively to the first and the second axis of the PCA performed on the seven environmental variables available for the 34 natural lakes. For the lake numbers, see the detailed list given in appendix 1.

\section{RÉSULTATS}

\subsection{Typologie physique des plans d'eau français}

La méthode des K-means permet d'identifier deux types de lacs naturels (Fig. 3 ) et trois types de retenues (Fig. 4). Les caractéristiques environnementales moyennes de ces cinq groupes de sites sont données dans le tableau I et représentées sur la figure 5 .

Le premier groupe de lacs naturels (LN1) est constitué de 24 sites d'altitude, plutôt petits, profonds et de forme plutôt arrondie (SLDF proche de 1). Le deuxième groupe de lacs naturels
(LN2) est constitué de 10 sites de très faible altitude, grands, peu profonds et peu découpés, mais dont les superficies peuvent être très différentes. Ces deux types de lacs naturels sont respectivement les plus froids et les plus chauds du jeu de données, les premiers pouvant avoir des températures moyennes hivernales négatives. Ils se distinguent clairement d'un point de vue géographique. Le premier groupe est constitué des plans d'eau des Alpes, du Jura et du Massif central, le deuxième des lacs des littoraux atlantique et méditerranéen.

Le premier groupe de réservoirs (RA1) est constitué de 20 plans d'eau 


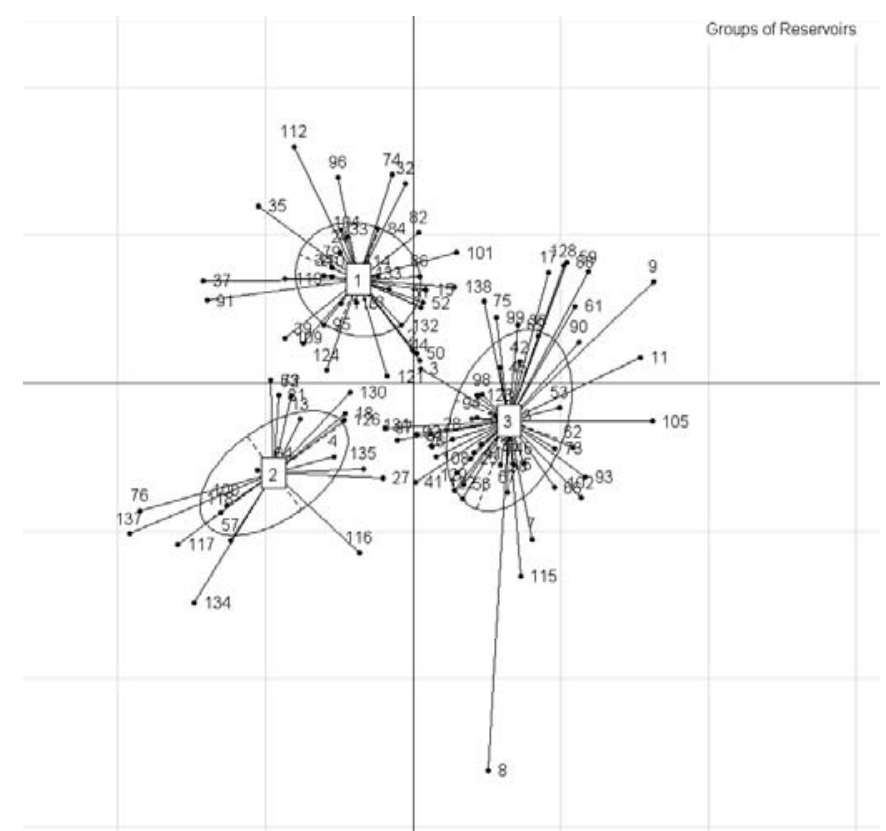

Fig. 4. Identification des trois groupes de retenues (1: groupe RA1; 2 : groupe RA2 ; 3 : groupe RA3) par la méthode des K-means - analyse sur 104 sites. Les axes horizontal et vertical correspondent, respectivement au premier et au deuxième axe de l'ACP réalisée sur les sept variables environnementales disponibles pour les 104 retenues. Pour les numéros des plans d'eau, voir la liste détaillée à l'annexe 1.

Fig. 4. Identification of three types of reservoirs (1: RA1 group; 2: RA2 group; 3: RA3 group) using the K-means approach. 104 reservoirs were analysed. Horizontal and vertical axes correspond, respectively to the first and the second axis of the PCA performed on the seven environmental variables available for the 104 reservoirs. For the lake numbers, see the detailed list given in appendix 1.

caractérisés par des altitudes intermédiaires, des surfaces et des profondeurs importantes, et des berges découpées (SLDF élevé). Le deuxième groupe (RA2) est constitué de 35 plans d'eau situés également à moyenne altitude mais de surface plutôt petite et de forme arrondie. Leur profondeur moyenne est intermédiaire. Le troisième groupe (RA3) rassemble 49 plans d'eau de faible altitude, de petite surface, à berge découpées, mais très peu profonds.

Les types RA1 et RA2 de retenues ne se distinguent pas forcément d'un point de vue géographique, ni du point de vue des températures, par contre le type RA3 regroupe majoritairement des retenues du bassin Loire/Bretagne, dont les températures hivernales et estivales sont moins contrastées que dans les deux autres types de retenues.

\subsection{Intensité des pressions}

\subsubsection{Analyse globale}

L'ACP réalisée sur l'ensemble des variables de pression des 138 plans 

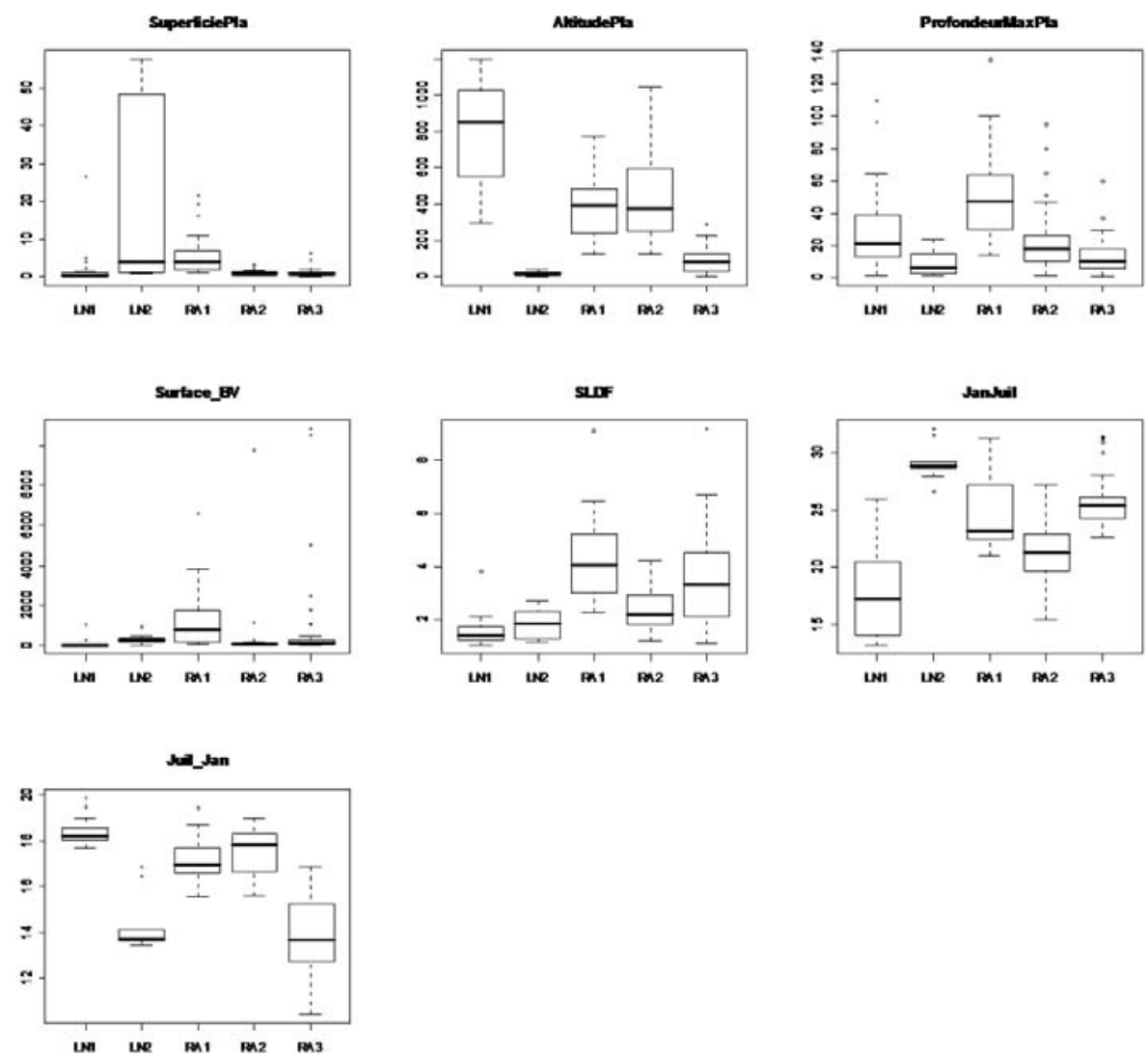

Fig. 5. Représentation des principales caractéristiques environnementales naturelles des cinq types de plans d'eau identifiés : surface du plan d'eau (SuperficiePla; en ha); altitude du plan d'eau (Altitude; en $\mathrm{m}$ ) ; profondeur maximale du plan d'eau (ProfondeurMaxPla; en $\mathrm{m}$ ); surface du bassin versant (Surface_BV; en $\mathrm{km}^{2}$ ); indice de développement des berges (SLDF; sans unité); somme des températures moyennes de l'air des mois de janvier et de juillet (JanJuil; en ${ }^{\circ} \mathrm{C}$ ); différence des températures moyennes de l'air entre le mois de juillet et le mois de janvier (Juil_Jan; en ${ }^{\circ} \mathrm{C}$ ).

Fig. 5. Boxplots of the main environmental characteristics for the five types of lakes: lake area (SuperficiePla; as ha); lake elevation (Altitude; as $\mathrm{m}$ ); lake maximal depth (ProfondeurMaxPla; as $\mathrm{m}$ ); drainage bassin area (Surface_BV; as $\mathrm{km}^{2}$ ); shoreline development factor (SLDF; S.I.); sum of January and July air mean temperatures (JanJuil; as ${ }^{\circ} \mathrm{C}$ ); difference between Jjuly and January air mean temperatures (Juil_Jan; as ${ }^{\circ} \mathrm{C}$ ).

d'eau permet d'identifier deux groupes de variables, les unes relatives à l'urbanisme, les autres en relation avec l'agriculture (Fig. 6). L'ACP réalisée sur les 5 variables de pressions agricoles
(Fig. 7a) montre que les 138 plans d'eau se répartissent le long du premier axe qui explique $73 \%$ de la variabilité. II porte la variable de transfert de phosphore et dans une moindre 


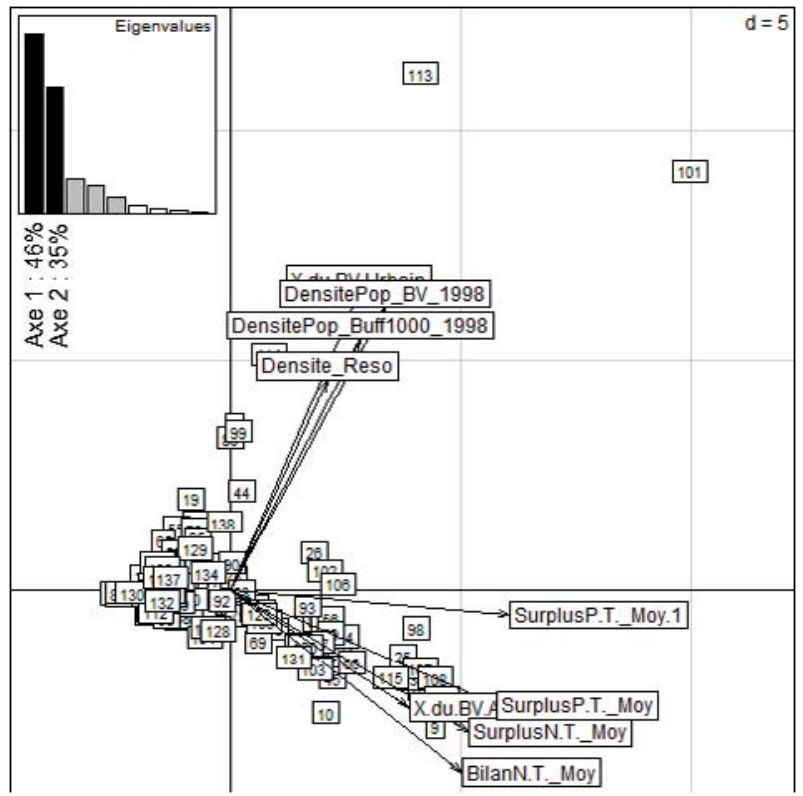

Fig. 6. Analyse en Composantes Principales (ACP) réalisée sur les neuf variables de pressions anthropiques : surface en zones urbaines sur le BV (X.du.BV.Urbain); surface en zones agricoles sur le BV (X.du.BV.Agri) ; densité de population sur le BV (DensitePop_BV_1998); densité de population sur un buffer de $1 \mathrm{~km}$ autour du lac (DensitePop_Buff1000_1998); densité de réseau de communication sur le BV (Densite_Reso) ; surplus en azote $\left(\mathrm{N}_{\text {total }}\right)$ et en phosphore $\left(\mathrm{P}_{\text {total }}\right)$ sur le BV (SurplusN.T._Moy et SurplusP.T._Moy) ; bilan en azote sur le BV (BilanN.T._Moy) ; aléa moyen d'érosion couplé aux surplus en $P_{\text {total }}$ du BV (SurplusP.T._Moy.1).

Fig. 6. Principal component analysis (PCA) carried out on the nine watershed pressure variables: urban areas within lake catchment (X.du.BV.Urbain); agriculture areas within lake catchment (X.du.BV.Agri); population density within lake catchment (DensitePop_BV_1998); population density into a $1 \mathrm{~km}$-buffer arround the lake (DensitePop_Buff1000_1998); network density within lake catchment (Densite_Reso); nitrogen $\left(\mathrm{N}_{\text {total }}\right)$ and phosphorus $\left(\mathrm{P}_{\text {total }}\right)$ surplus within lake catchment (SurplusN.T._Moy et SurplusP.T._Moy); nitrogen balance within lake catchment (BilanN.T._Moy) and phosphorus balance multiplied by the mean value of the lake's watershed soil erosion (SurplusP.T._Moy.1).

mesure les variables bilan et surplus d'azote total. Globalement, les sites peu impactés par ces variables sont plus nombreux que les plans d'eau avec des valeurs fortes. Néanmoins, toute la gamme des pressions anthropiques est observée.

Les premier et deuxième axes de l'ACP, réalisée sur les 4 variables de pressions urbaines disponibles pour les 138 plans d'eau, expliquent respectivement $70 \%$ et $17 \%$ de la variabilité des sites (Fig. 7b). Le premier axe est fortement tiré par deux sites très impactés [retenues de Grand-Large $\left(n^{\circ} 101\right.$, Fig. 7b) et de Torcy-Neuf $\left(n^{\circ} 113\right.$, Fig. $\left.7 b\right)$ avec, respectivement, $42 \%$ et $55 \%$ du BV urbanisé], qui s'opposent à l'ensemble des autres plans d'eau. Un lac [Nantua, $\mathrm{n}^{\circ} 80$, 


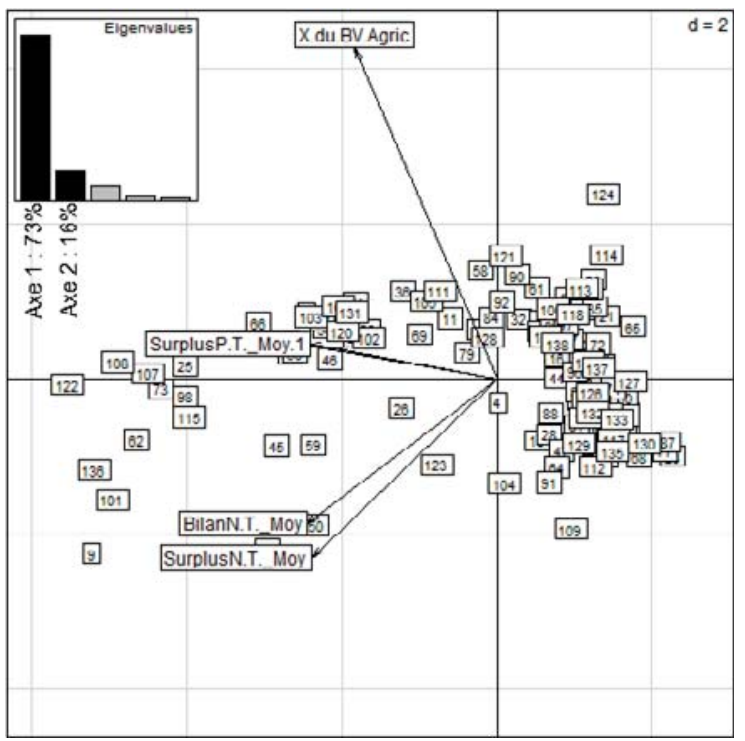

(a)

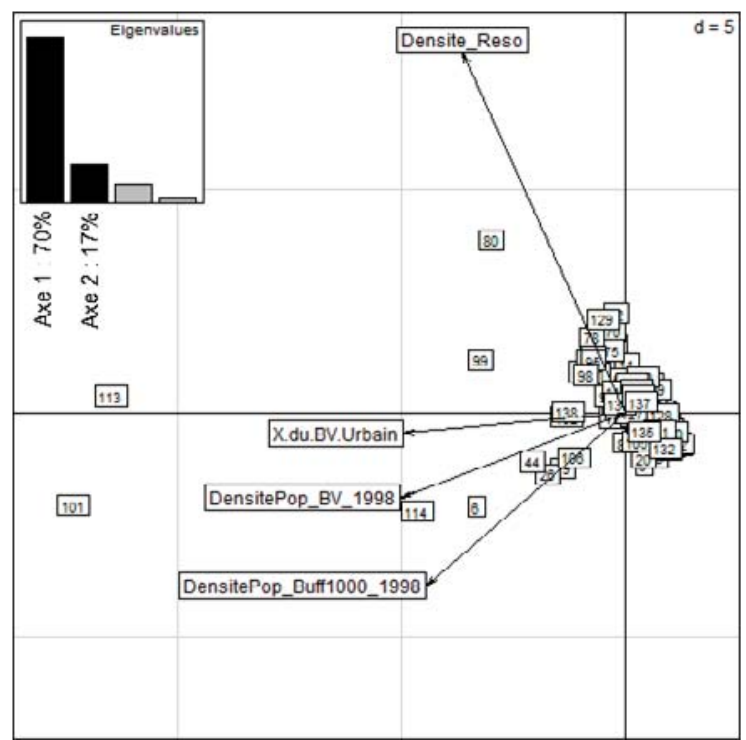

(b)

Fig. 7. Analyse en Composantes Principales (ACP) réalisée sur les cinq variables de pressions agricoles (a), et les quatre variables de pressions urbaines (b).

Fig. 7. Principal component analysis (PCA) carried out on (a) the five agricultural pressure variables, and (b) the four urban pressure variables. 

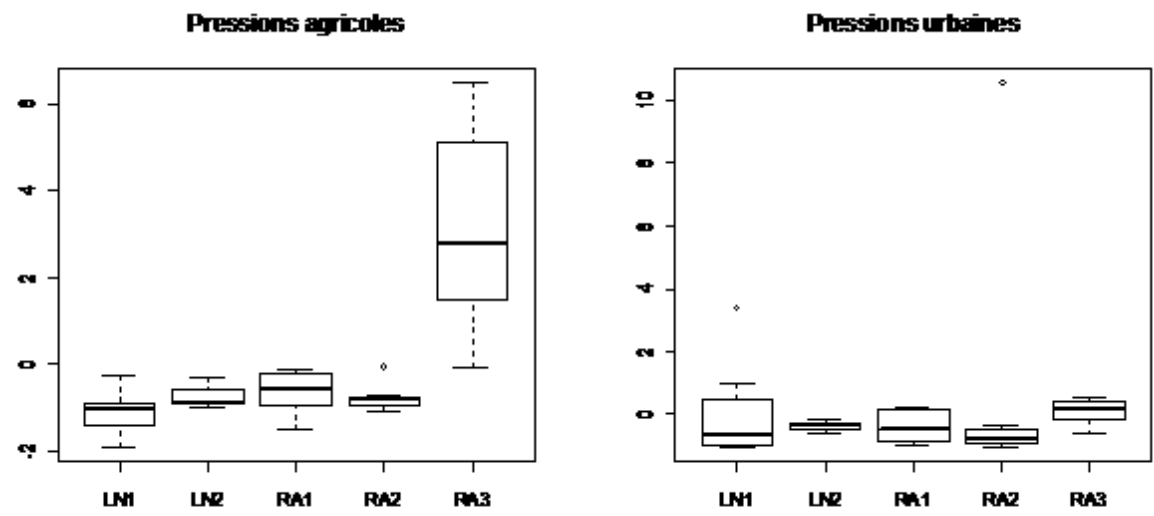

(a)

(b)

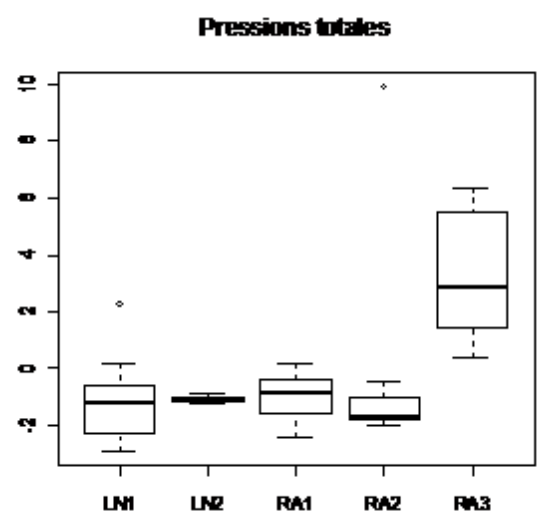

(c)

Fig. 8. Représentation des trois indices synthétiques de pressions anthropiques pour chacun des cinq types de plans d'eau identifiés : indice synthétique des pressions agricoles (a), indice synthétique des pressions urbaines (b), indice de pression global (c).

Fig. 8. Boxplots of the three synthetic anthropogenic pressure indices for each of the five types of lakes: (a) agricultural pressure synthetic index, (b) urban pressure synthetic index, (c) global pressure index.

Fig. 7b] se distingue sur cet axe par son important réseau de communication sur son BV.

\subsubsection{Analyse par types}

L'analyse par types, menée sur les 44 plans d'eau présentant des don- nées poissons, indique que l'intensité des pressions agricoles est comparable entre les groupes LN1 et LN2 des lacs naturels (respectivement, $\mathrm{n}=16$ et $n=3$ ) et RA1 et RA2 des retenues (respectivement, $n=8$ et $n=7$ ) (Fig. 8a). Au sein de ces 4 groupes, les intensités de pressions sont beaucoup plus 
faibles que dans le groupe 3 des retenues (RA3; $n=10$ ), qui se démarque très nettement. Dans ce dernier type, tous les sites sont plus impactés que ceux des autres types.

Les pressions urbaines sont aussi globalement assez faibles sur l'ensemble des sites, quel que soit le groupe de plans d'eau considéré (Fig. 8b). On relève pourtant des valeurs très élevées de pressions urbaines pour deux sites, à savoir TorcyNeuf et Annecy, classés respectivement dans le groupe 2 des retenues (RA2) et dans le groupe 1 des lacs naturels (LN1).

Le groupe 3 des retenues (RA3) qui se distingue par de fortes pressions agricoles, présente également un indice de pressions globales élevé. De même, les deux sites fortement marqués par les pressions urbaines sont très impactés lorsque l'on considère les pressions totales (Fig. 8c).

\subsection{Calcul des métriques piscicoles et réponse aux pressions}

Vingt neuf espèces de poissons ont été recensées sur les seize lacs naturels du groupe LN1 et les 10 retenues du groupe RA3. Les caractéristiques des espèces piscicoles capturées sur ces sites sont listées dans le tableau II. La perche franche (Perca fluviatilis) et le gardon (Rutilus rutilus) sont les plus fréquents quel que soit le type de milieu et la plupart des espèces sont communes aux deux types de systèmes. Inversement, dix espèces ne sont présentes que dans les lacs naturels. Parmi ces espèces, on compte trois salmonidés (Salmo trutta fario, Salvelinius alpinus et Salvelinius namaycush). La bouvière (Rhodeus ama- rus) et le mulet porc (Liza ramada) n'ont été capturés que dans des retenues.

Dans les retenues du groupe 3, aucune des métriques calculées ne répond à l'indice de pression urbaine. Par contre trois métriques répondent positivement aux pressions agricoles qui s'exercent sur le bassin versant (Fig. 9, Tab. IV) : il s'agit de la richesse en espèces phytophiles et des densités de poissons, exprimées en biomasse ou en effectifs. La richesse en espèces phytophiles est la seule métrique répondant positivement à l'indice de pression globale.

Sur les lacs naturels du type un, cinq métriques répondent positivement aux pressions urbaines. II s'agit des richesses spécifiques totales, en espèces natives, en espèces tolérantes, en espèces omnivores-généralistes et en espèces planctivores (Tab. IV). Cependant, les corrélations observées sur les richesses totales et en espèce natives sont fortement tirées par un point (Fig. 10). Les richesses en espèces tolérantes, planctivores et omnivores-généralistes sont aussi positivement corrélées à l'indice de pression totale. La richesse en espèces tolérantes est la seule métrique qui répond aux trois indices de pression.

\section{DISCUSSION ET CONCLUSIONS}

Ce travail constitue une étape exploratoire de développement d'un indicateur poisson pour les plans d'eau français, réalisée à partir des données nationales disponibles fin 2008.

La typologie effectuée ici prend en compte des variables environnementales connues pour expliquer la 
Rsp $=0.65$

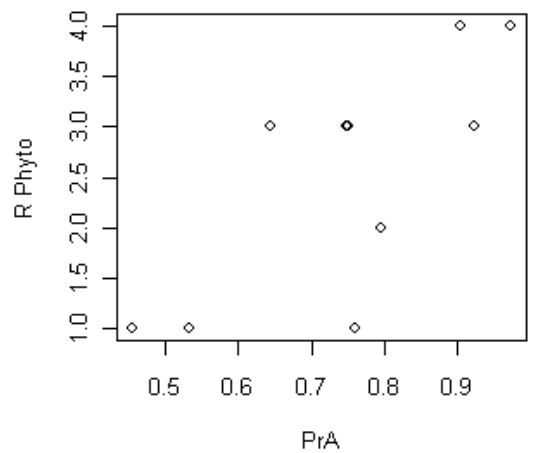

$\mathrm{Rsp}=0.65$

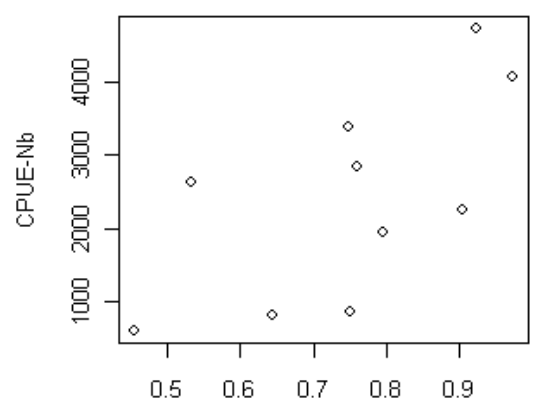

$\operatorname{PrA}$
$\mathrm{Rsp}=0.65$

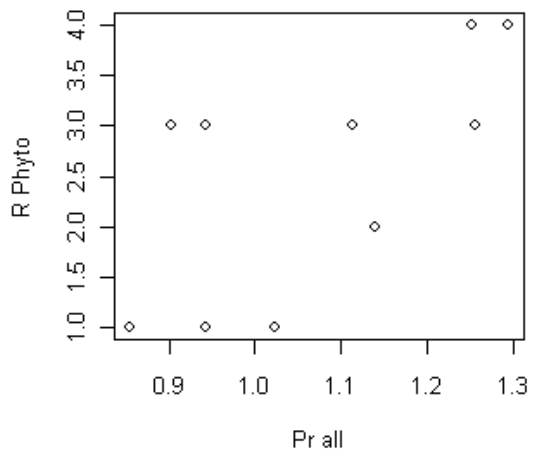

$\mathrm{Rsp}=0.72$

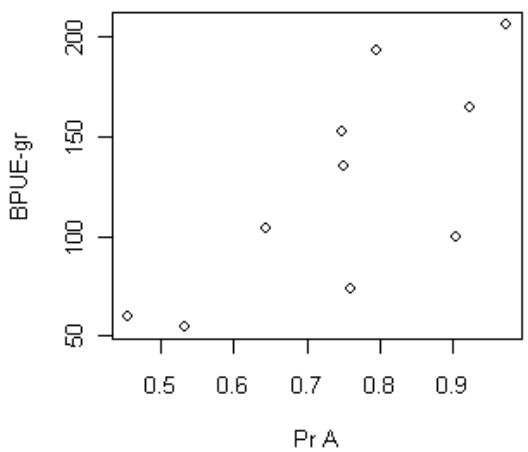

Fig. 9. Représentations graphiques des corrélations de Spearman (Rsp : valeur du coefficient de corrélation) entre les métriques sélectionnées dans le type RA3 des retenues et les indices synthétiques de pressions, avec $\operatorname{Pr} \mathrm{A}$ : indice synthétique des pressions agricoles; $\operatorname{Pr}$ all : indice de pression global; R Phyto : nombre d'espèces de poissons phytophiles; CPUE-Nb : captures par unité d'effort de pêche et BPUE-gr : biomasse par unité d'effort de pêche.

Fig. 9. Scatter plots of Spearman's correlations (Rsp: value of the correlation coefficient) between selected fish based metrics for reservoirs of the RA3 group and synthetic anthropogenic pressure indices, with $\operatorname{Pr} A$ : agricultural pressure synthetic index; $\operatorname{Pr}$ all: global pressure index; R Phyto: number of phytophilic species; CPUE-Nb: catch per unit effort and BPUE-gr: biomass per unit effort.

variabilité des communautés de poissons. Néanmoins, d'autres variables importantes, vis-à-vis des propriétés physico-chimiques de l'eau, telles que la géologie et l'hydrologie auraient dû être prises en compte (Rowan et al., 2003; Winfield \& Durie, 2004), mais elles ne sont pas disponibles pour l'ensemble des sites. La typologie regroupe donc des milieux encore hétérogènes par certains aspects environnementaux.

Sur les lacs naturels, la typologie a été effectuée sur les données de 
Tableau IV. Corrélation de Spearman entre les métriques et les pressions au sein de chaque type de plans d'eau : * significatif à $p<0,05$.

Table IV. Spearman's correlation coefficients between fish based metrics and anthropogenic pressures, in each type of lakes: * significant at 0.05 level.

\begin{tabular}{lccc}
\hline Métriques & \multicolumn{3}{c}{ Indices synthétiques de pressions } \\
\cline { 2 - 4 } & urbaines & agricoles & urbaines + agricoles \\
\hline Groupe 3 des retenues $(\mathrm{n}=10)$ & & & \\
Richesse en phytophiles stricts & $-0,27$ & $0,65^{*}$ & $0,65^{*}$ \\
CPUE-Nb & $-0,50$ & $0,65^{*}$ & 0,41 \\
BPUE_gr & $-0,53$ & $0,72^{*}$ & 0,52 \\
\hline Groupe 1 des lacs naturels $(\mathrm{n}=16)$ & & & \\
Richesse spécifique & $0,56^{*}$ & 0,36 & 0,49 \\
Richesse en espèces natives & $0,53^{*}$ & 0,32 & 0,45 \\
Richesse en espèces tolérantes & $0,59^{*}$ & $0,6^{*}$ & $0,62^{*}$ \\
Richesse en espèces planctivores & $0,56^{*}$ & 0,43 & $0,50^{*}$ \\
Richesse en espèces omnivores - & $0,57^{*}$ & 0,49 & $0,58^{*}$ \\
généralistes & & & \\
\hline
\end{tabular}

34 plans d'eau, ce qui représente la moitié des milieux concernés par la DCE (71 lacs, R. Lallemand, communication personnelle) et deux types de milieux ont été identifiés. Ainsi, quelle que soit la représentativité de nos milieux, il est évident que scinder les lacs français en groupes homogènes va nous amener à des types avec des effectifs très faibles, et/ou à des types au sein desquels va demeurer une forte variabilité environnementale, source de variabilité naturelle des communautés piscicoles.

Pour les retenues, trois types de milieux ont été identifiés avec notre jeu de données qui représente moins d'un tiers de l'ensemble des milieux artificiels français. En supposant que notre échantillonnage soit représentatif de la diversité des sites nationaux, il est vraisemblable que les retenues non considérées ici se répartissent au sein des types existants.
Dans le cas contraire, quelques nouveaux types pourraient être identifiés. Néanmoins, compte tenu que cette typologie n'intègre pas de variable hydrologique, telle que le temps de renouvellement des masses d'eau par exemple, il est probable que la variabilité intra-type demeure forte.

Il faut également noter qu'en raison de la prise en compte de variables environnementales mieux adaptées à l'ichtyofaune, dans la méthode utilisée ici pour identifier des types plus homogènes, ces derniers ne recoupent pas parfaitement ceux identifiés dans la typologie nationale (Ministère de l'Écologie et du Développement Durable, 2005).

Les milieux ont ensuite été caractérisés par les pressions (ou forces motrices, voir IMPRESS, 2002) qui s'exercent sur les bassins versants et qui sont les seules données homogènes disponibles pour l'ensemble des 
$\mathrm{Rsp}=0.56$

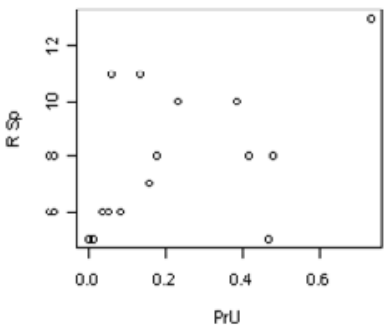

$\mathrm{Rsp}=0.6$

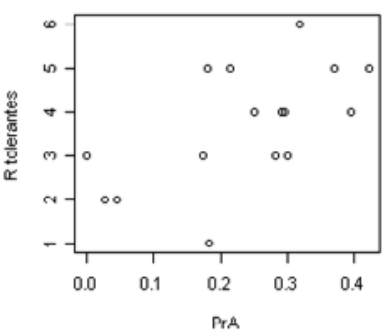

$\mathrm{Rsp}=0.5$

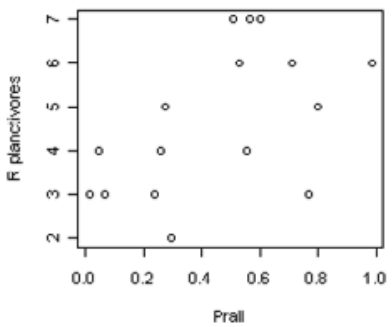

Rsp $=0.53$

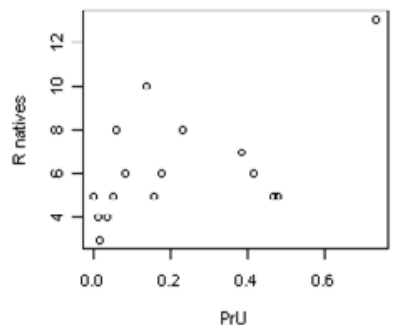

$\mathrm{Rsp}=0.62$

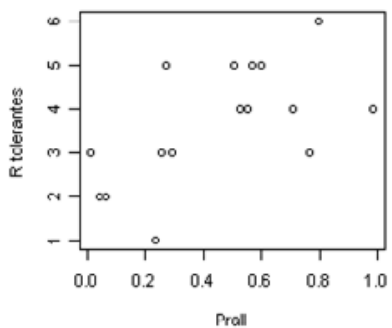

$\mathrm{Rsp}=0.57$

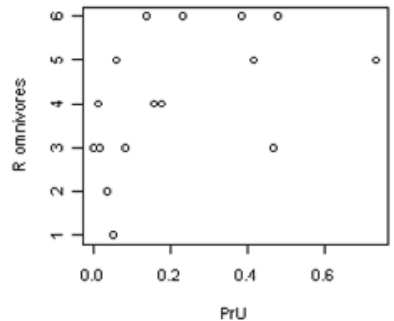

$\mathrm{Rsp}=0.59$

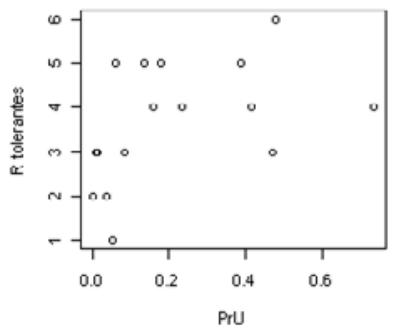

$\mathrm{Rsp}=0.56$

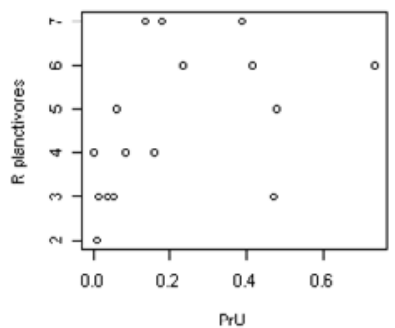

$\mathrm{Rsp}=0.58$

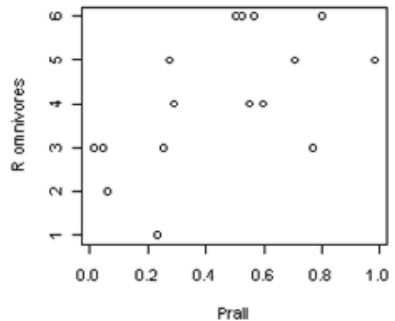

Fig. 10. Représentations graphiques des corrélations de Spearman (Rsp : valeur du coefficient de corrélation) entre les métriques sélectionnées dans le type LN1 des lacs naturels et les indices synthétiques de pressions, avec $\operatorname{Pr} A$ : indice synthétique des pressions agricoles; $\operatorname{Pr} U$ : indice synthétique des pressions urbaines ; $\mathrm{Pr}$ all : indice de pression global; $\mathrm{R} \mathrm{Sp}$ : richesse spécifique; $\mathrm{R}$ natives : nombre d'espèces de poissons natifs; $R$ tolerantes : nombre d'espèces de poissons tolérants ; $R$ planctivores : nombre d'espèces de poissons planctivores et $R$ omnivores : nombre d'espèces de poissons omnivores.

Fig. 10. Scatter plots of Spearman's correlations (Rsp: value of the correlation coefficient) between selected fish based metrics for natural lakes of the LN1 group and synthetic anthropogenic pressure indices, with $\operatorname{Pr} \mathrm{A}$ : agricultural pressure synthetic index; $\operatorname{Pr} \mathrm{U}$ : urban pressure synthetic index; $\operatorname{Pr}$ all: global pressure index; R Sp: species richness; R natives: number of native species; $R$ tolerantes: number of tolerant species; $R$ planctivores: number of planctivorous species and R omnivores: number of omnivorous species. 
sites. Ainsi, les données de pressions analysées reflètent bien l'ensemble des sources de pollution chimique, et, partiellement, les pressions s'exerçant sur la morphologie des lacs. En effet, la densité de population et la densité du réseau routier sont de bons indicateurs de la fréquentation des milieux (Forman \& Alexander, 1998; Jones et al., 2000 ; Pitt et al., 1995).

II faut noter que la variabilité des pressions agricoles et urbaines au sein de la plupart des types est très faible. De plus, elle n'est pas comparable sur l'ensemble des types. Dans quelques cas (types RA2 et LN1), des pressions urbaines très fortes sont mesurées sur quelques sites isolés, sans pour autant que la gamme de pressions soit couverte de manière homogène par l'ensemble des plans d'eau. Si elles devaient être confirmées, ces distributions hétérogènes de sites plus ou moins impactés, au sein des types et entre les types, pourraient poser des problèmes quant à la définition de seuils homogènes de qualité des métriques à l'échelle nationale.

Le développement de bioindicateurs suppose que l'on dispose de mesures sur des sites pas ou peu impactés, ces sites servant de base de référence aux métriques sélectionnées pour leur réponse aux pressions (Noble et al., 2007). Cette approche typologique suppose donc l'établissement de conditions de référence au sein de chaque type. Nous avons montré que, comparativement aux autres, le type 3 des retenues ne présentait pas de site peu impacté; par conséquent, nous constatons que ceci constitue une limite au développement d'un indice poisson.
Une condition supplémentaire à la sélection de métriques réside dans l'obtention d'une gamme assez large et complète de pressions. Or, nous avons montré que ceci n'était le cas pour aucun des types et aucune des pressions, hormis pour les pressions agricoles du type RA3, type pour lequel se posait le problème de l'absence de référence.

De plus, la gamme de variations des pressions anthropiques doit être à peu près identique au sein de chaque type, sans quoi le système de notation ne pourra pas être harmonisé. Même si ces gammes de variations des pressions anthropiques se chevauchent souvent entre types, cette condition n'est pas remplie, notamment si l'on considère les quelques milieux très perturbés du type 1 des lacs naturels et du type 2 des retenues.

D'autres pressions s'exercent directement sur les systèmes et sont à même d'influencer l'ichtyofaune. C'est le cas par exemple des variations de niveau d'eau (Argillier et al., 2004; Poirel et al., 1996). L'exercice conduit ici devra donc être élargi à l'ensemble des pressions, en particulier à la mesure de l'altération de l'hydromorphologie des systèmes et des usages associés.

L'analyse de la sélection des métriques repose sur les observations réalisées sur les types de milieux les mieux représentés. Malgré cela, le nombre de sites reste très faible, en particulier sur les retenues. Sur ces types avec un petit nombre d'échantillons, les résultats sont très dépendants des données. Cet exercice de sélection de métriques doit donc être considéré comme une étape exploratoire. 
Nous avons montré qu'il était possible d'identifier des métriques élaborées à partir des caractéristiques de communautés piscicoles, répondant aux pressions anthropiques. Finalement, au sein de chacun des deux groupes de plans d'eau étudiés, la plupart des corrélations ne sont observées qu'avec des pressions dont la gamme de variation est la plus forte, à savoir, les pressions agricoles dans le type RA3 et les pressions urbaines dans le type LN1. Selon la distribution des gammes de pressions au sein des types, l'agrégation des pressions n'est donc pas forcément une étape intéressante pour l'élaboration des indices.

Dans le groupe des retenues, toutes les métriques sont positivement corrélées à l'agriculture. La réponse des densités de poissons (en effectif ou en biomasse) aux pressions est assez contrastée selon les études. Une diminution des densités piscicoles avec l'augmentation des pressions a été reportée sur les retenues des États-Unis (Hickman \& McDonough, 1996) ; inversement, sur les lacs naturels d'Europe, c'est une augmentation qui est mesurée (Pedron et al., 2010), conformément à ce que nous observons ici. L'augmentation du nombre d'espèces phytophiles strictes avec l'agriculture n'a pas été documentée. On aurait pu s'attendre à une diminution du nombre d'espèces, dont la reproduction est liée à la qualité des habitats littoraux, avec l'augmentation des pressions du bassin versant. Ce résultat met en avant la limite du jeu de données dans ce type d'approche.

Sur les lacs naturels, le nombre de métriques potentiellement intéressantes est beaucoup plus important que sur les retenues. Toutefois, parmi les neuf corrélations significatives, celles mesurées entre la richesse spécifique totale ou la richesse spécifique en espèces natives et la pression urbaine sont douteuses du fait de la distribution des valeurs mesurées. Inversement, l'analyse graphique des sept autres corrélations renforce les résultats issus des analyses statistiques.

L'inclusion de métriques liées à la tolérance est très fréquente dans les indices poisson (Jennings et al., 1999; Whittier, 1999) et la relation positive observée ici avec toutes les pressions va dans le sens attendu selon la littérature. Bien que très répandue, l'utilisation de cette métrique est discutable; en effet, la bonne réponse aux pressions peut être attribuée au mode de construction de cette métrique, la tolérance des espèces étant définie, précisément, à partir de leur fréquence d'observation en milieux perturbés!

La réponse des espèces omnivoresgénéralistes aux pressions est également bien documentée et les résultats obtenus sont en cohérence avec la plupart des observations (Drake \& Pereira, 2002 ; Schulz et al., 1999).

Pour conclure, ces résultats mettent en avant un certain nombre de limites à l'approche typologique afin de développer un indice harmonisé au niveau national. Le faible nombre de lacs naturels par type limite la pertinence des corrélations potentielles entre les métriques et les pressions. De plus, les différences inter- et intra-types de gradients de pressions rendent difficilement envisageable une approche harmonisée au niveau national. II conviendra de vérifier ces limites lorsque plus de données seront disponibles. Si elles devaient être 
confirmées, d'autres approches, notamment par modélisation sur l'ensemble des sites, devraient être envisagées.

Au contraire, lorsque le jeu de données est significatif, il est possible de définir certaines métriques relatives à l'ichtyofaune répondant de manière cohérente à des pressions d'origines agricole ou urbaine. Ces premiers résultats sont donc très encourageants quant à la possibilité d'utiliser l'ichtyofaune pour rendre compte de perturbations anthropiques, ceci malgré l'idée fréquemment avancée que ces communautés piscicoles sont très manipulées par l'Homme et qu'elles reflètent donc peu les conditions environnementales.

\section{REMERCIEMENTS}

Les auteurs remercient $\mathrm{H}$. Persat (CNRS), N. Poulet (ONEMA) et O. Schlumberger (Ministère de l'Écologie, du Développement durable, des Transports et du Logement), qui ont vérifié le tableau des traits fonctionnels piscicoles. Nous remercions aussi G. Legall (PÖYRY Environment); V. Antoni (INRA Orléans); les équipes du BRGM d'Orléans; toutes les personnes de I'INERIS ayant collaboré à ce projet ; C. Heyd, S.Alleaume et J.-M. Helmer (CEMAGREF); O. Coulon, L. Navarro et L. Fournier (Agences de l'Eau); ainsi que V. Verneaux (Université de Franche-Comté) pour leur aide lors de l'acquisition de données et l'utilisation de données SIG. Les auteurs tiennent aussi à remercier spécialement M. Gevrey pour ses précieux conseils en matière de statistiques, ainsi que pour sa contribution aux illustrations. Enfin nous remercions tout particulièrement $\mathrm{J}$. Veslot, A. Brind'Amour et $D$. Pont pour leurs idées et leurs précieux commentaires lors de cette étude. Cette étude a été financée par l'Office National de l'Eau et des Milieux Aquatiques (ONEMA).

\section{RÉFÉRENCES BIBLIOGRAPHIQUES}

Argillier C., Pronier O. \& Irz P. 2002. Approche typologique des peuplements piscicoles lacustres Français. I. Les communautés des plans d'eau d'altitude supérieure à $1500 \mathrm{~m}$. Bulletin Français de Pêche et de Pisciculture 365/366 : 373-387.

Argillier C., Poulet N. \& Irz P. 2004. Effect of meteorological conditions and water level fluctuations on the year-class strength of pikeperch (Sander lucioperca L.) and perch (Perca fluviatilis L.) in a French reservoir. Ecohydrology \& Hydrobiology 4 (4) : 441-448.

Balon E.K. 1975. Reproductive guilds of fishes: a proposal and definition. Journal of the Fisheries Research Board of Canada 32 : 821-864.

Benichou P. \& Le Breton O. 1987. Prise en compte de la topographie pour la cartographie des champs pluviométriques statistiques. La Météorologie 7 (19) : 23-34.

Bruslé J. \& Quignard J.P. 2001. Biologie des poissons d'eau douce européens. Lavoisier Tec \& Doc. Paris.

Carpenter S.R., Caraco N.F., Correll D.L., Howarth R.W., Sharpley A.N. \& Smith V.H. 1998. Nonpoint pollution of surface waters with phosphorus and nitrogen. Ecological Applications 8 (3) : 559-568.

CEN. 2005. Water Quality - Sampling of fish with multi-mesh gillnets. $26 \mathrm{pp}$.

Coale F.J. 2000. The Maryland Phosphorus Site Index (PSI) technical user's guide. In Soil Fertility Management 
Information Series, SFM-7. Maryland Extension Service.

Communauté européenne. 2000. Directive 2000/60/CE du Parlement Européen et du Conseil du 23 octobre 2000. Journal Officiel des Communautés Européennes : L327.

De Bortoli J. \& Argillier C. 2008. Définition des conditions de référence et des limites des classes d'état sur la base d'une approche pressions/impacts - Plans d'eau - Paramètre chlorophylle-a. 51.

Drake M.T. \& Pereira D.L. 2002. Development of a fish-based index of biotic integrity for small inland lakes in central Minnesota. North American Journal of Fisheries Management, 22 (4) : 1105-1123.

Eadie J.M. \& Keast A. 1984. Resource heterogeneity and fish species diversity in lakes. Canadian Journal of Zoology, 62 (9) : 1689-1695.

Forman R.T.T. \& Alexander L.E. 1998. Roads and their major ecological effects. Annual Review of Ecology and Systematics, $29: 207-231$.

Hawkins C.P., Norris R.H., Gerritsen J., Hughes R.M., Jackson S.K., Johnson R.K. \& Stevenson R.J. 2000. Evaluation of the use of landscape classifications for the prediction of freshwater biota: synthesis and recommendations. Journal of the North American Benthological Society, 19 (3) : 541-556.

Hickman G.D. \& McDonough T.A. 1996. Assessing the reservoir fish assemblage index: A potential measure of reservoir quality. In Multidimensional approaches to reservoir fisheries management L.E. Miranda and D.R. DeVries Eds., pp. 85-97.

Ihaka R. \& Gentleman R. 1996. A language for data analysis and graphics. Journal of Computational and Graphical Statistics 5 : 299-314.

IMPRESS. 2002. Guidance for the analysis of pressures and impacts in accordance with the Water Framework Directive. Common Implementation Strategy Working Group 2.1. 156 p.

Jennings M.J., Lyons J., Emmons E.E., Hatzenbeler G.R., Bozek M.A.,
Simonson T.D., Beard T.D., Jr \& Fago D. 1999. Toward the development of an index of biotic integrity for inland lakes in Wisconsin. In Assessing the sustainability and biological integrity of water resource quality using fish communities, T.P. Simon Ed., pp. 541-562.

Jones J.A., Swanson F.J., Wemple B.C. \& Snyder K.U. 2000. Effects of roads on hydrology, geomorphology, and disturbance patches in stream networks. Conservation Biology 14 (1) : 76-85.

Keith P. \& Allardi J. 2001. Atlas des poissons d'eau douce de France. In Patrimoines Naturels 387.

MacQuenn J.B. 1967. Some Methods for classification and Analysis of Multivariate Observations. University of California Press. Berkeley.

Ministère de l'Écologie et du Développement Durable. 2005. Circulaire DCE 2005/11 relative à la typologie nationale des eaux de surface (cours d'eau, plans d'eau, eau de transition et eaux côtières) en application de la directive 2000/60/DCE du 23 octobre 2000 du Parlement et du Conseil établissant un cadre pour une politique communautaire dans le domaine de l'eau. In Bulletin Officiel 14.

Noble R.A.A., Cowx I.G., Goffaux D. \& Kestemont P. 2007. Assessing the health of European rivers using functional ecological guilds of fish communities: standardising species classification and approaches to metric selection. Fisheries Management and Ecology 14 (6) : 381-392.

Oberdorff T., Pont D., Hugueny B. \& Chessel D. 2001. A probabilistic model characterizing fish assemblages of French rivers: a framework for environmental assessment. Freshwater Biology 46 : 399-415.

Oberdorff T., Pont D., Hugueny B. \& Porcher J.-P. 2002. Development and validation of a fish-based index for the assessment of 'river health' in France. Freshwater Biology 47 (9) : 1720-1734.

Pedron S., De Bortoli J. \& Argillier C. 2010. Deliverable 3.4-4: Fish indicators for 
ecological status assessment of lakes affected by eutrophication and hydromorphological pressures. WISER Ed.

Pitt R., Field R., Lalor M. \& Brown M. 1995. Urban Stormwater Toxic Pollutants Assessment, Sources, and Treatability. Water Environment Research 67 (3) : 260-275.

Plafkin J.L., Barbour M.T., Porter K.D., Gross S.K. \& Hughes R.M. 1989. Rapid bioassessment protocols for use in streams and rivers.

Poirel A., Merle G., Salençon M.J. \& Travade F. 1996. Gestion hydraulique et ressources piscicoles dans les retenues hydroélectriques.

Pont D., Hugueny B., Beier U., Goffaux D., Noble R., Rogers C., Roset N. \& Schmutz S. 2006. Assessing river biotic condition at a continental scale: a European approch using functional metrics and fish assemblages. Journal of Applied Ecology 43 : 70-80.

Pont D., Hugueny B. \& Rogers C. 2007. Development of a fish-based index for the assessment of river health in Europe: the European Fish Index. Fisheries Management and Ecology 14 (6) : 427-440.

R Development Core Team. 2008. A language and environment for statistical computing. R Foundation for Statistical Computing. Vienna, Austria. Available at: http://www.R-project.org.
Rowan J.S., Bragg O.M., Duck R.W. \& Black A.R. 2003. Development of a technique for lake habitat survey (LHS): Scoping study. Final report. 55.

Schmutz S., Melcher A., Frangez C., Haidvogl G., Beier U., Böhmer J., Breine J., Simoens I., Caiola N. \& De Sostoa A. 2007. Spatially based methods to assess the ecological status of riverine fish assemblages in European ecoregions. Fisheries Management and Ecology 14 (6) : 441452.

Schulz E.J., Hoyer M.V. \& Canfield D.E., Jr. 1999. An Index of Biotic Integrity: A test with limnological and fish data from sixty Florida lakes. Transactions of the American Fisheries Society 128 (4) : 564-577.

Schupp D.H. 1992. An ecological classification of Minnesota lakes with associated fish communities. In Investigational Report 41.

Whittier T.R. 1999. Development of IBI metrics for lakes in Southern New England. In Assessing the sustainability and biological integrity of water resource quality using fish communities T.P. Simon Ed., pp. 563-584.

Winfield I.J. \& Durie N.C. 2004. Fish introductions and their management in the English Lake District. Fisheries Management and Ecology 11 (3-4) : 195-201. 


\section{ANNEXE 1}

Liste des 138 plans d'eau français, pour lesquels des données environnementales sont disponibles, analysés dans cette étude. Ce tableau donne le nom du plan d'eau (Nom_Lac), le groupe typologique auquel le plan d'eau appartient (Grp_Lac; LNX : lacs naturel du type X et RAY : réservoir du type $Y$ ) et le numéro du plan d'eau sur les figures (Num_Lac). Les 44 plans d'eau, pour lesquels des données poissons sont également disponibles, apparaissent en gras dans la liste.

\section{APPENDIX 1}

List of the 138 lakes studied for which environmental data are available. This table gives the lake name (Nom_Lac), the typological group of the lake (Grp_Lac ; LNX : natural lake belonging to group $X$ and $R A Y$ : reservoir belonging to group $Y$ ) and the lake number on the figures (Num_Lac). The 44 lakes for which fish data are also available are in bold in the list.

\begin{tabular}{|l|c|c|}
\hline Nom_Lac & Grp_Lac & Num_Lac \\
\hline Abbaye (l' ) & LN1 & 1 \\
\hline Annecy (lac d') & LN1 & $\mathbf{6}$ \\
\hline Aydat (lac d') & LN1 & $\mathbf{1 4}$ \\
\hline Barterand (lac de ) & LN1 & $\mathbf{1 6}$ \\
\hline Bordes (lac des ) & LN1 & $\mathbf{2 1}$ \\
\hline Bouchet (lac du ) & LN1 & $\mathbf{2 2}$ \\
\hline Bourdouze (lac de ) & LN1 & $\mathbf{2 3}$ \\
\hline Cassiere (lac de la ) & LN1 & $\mathbf{2 9}$ \\
\hline Chalain (lac de ) & LN1 & $\mathbf{3 8}$ \\
\hline Chambon (lac ) & LN1 & $\mathbf{4 0}$ \\
\hline Etival (grand lac ) & LN1 & $\mathbf{4 9}$ \\
\hline Clairvaux (Grand lac) & LN1 & 55 \\
\hline Issarlès (lac d' ) & LN1 & $\mathbf{6 0}$ \\
\hline Chaillexon (lac de ) & LN1 & $\mathbf{6 5}$ \\
\hline Grand maclu (lac du ) & LN1 & $\mathbf{6 8}$ \\
\hline Pierre-châtel (lac de ) & LN1 & $\mathbf{7 0}$ \\
\hline Rousses (lac des ) & LN1 & 71 \\
\hline Montcineyre (lac de ) & LN1 & $\mathbf{7 7}$ \\
\hline Nantua (lac de ) & LN1 & 80 \\
\hline Paladru & LN1 & 83 \\
\hline Pavin (lac ) & LN1 & $\mathbf{8 7}$ \\
\hline Petichet & LN1 & 89 \\
\hline Saint Point & LN1 & 127 \\
\hline Sylans (lac de ) & LN1 & 129 \\
\hline Aulnes (étang des) & LN2 & 10 \\
\hline Aureilhan (étang d' ) & LN2 & 12 \\
\hline Biscarrosse (petit étang de ) & LN2 & 19 \\
\hline Blanc (étang du ) & LN2 & $\mathbf{2 0}$ \\
\hline
\end{tabular}




\begin{tabular}{|c|c|c|}
\hline Cazaux (étang de ) & LN2 & 30 \\
\hline Carcans-Hourtin (étang de ) & LN2 & 47 \\
\hline Entressen (d') & LN2 & 48 \\
\hline Grand-lieu & LN2 & 54 \\
\hline Parentis & LN2 & 85 \\
\hline Soustons (étang de ) & LN2 & 125 \\
\hline Age (l') & RA1 & 2 \\
\hline Bairon (étang de ) & RA1 & 15 \\
\hline Saint-agnan (barrage de ) & RA1 & 24 \\
\hline Cercey (barrage de ) & RA1 & 32 \\
\hline Chamboux (retenue de ) & RA1 & 33 \\
\hline Chammet (retenue du ) & RA1 & 34 \\
\hline Châtelot (du ) & RA1 & 35 \\
\hline Chambon (lac du ) & RA1 & 37 \\
\hline Chaumeçon (barrage de ) & RA1 & 39 \\
\hline Drapeau (du ) & RA1 & 44 \\
\hline Val Joly (lac du ) & RA1 & 50 \\
\hline Feyt (lac de ) & RA1 & 51 \\
\hline Filleit (barrage du ) & RA1 & 52 \\
\hline Méouze (étang de ) & RA1 & 74 \\
\hline Montaubry (barrage de ) & RA1 & 78 \\
\hline Mouche (barrage de la ) & RA1 & 79 \\
\hline Ospédale (l') & RA1 & 82 \\
\hline Panthier (lac de ) & RA1 & 84 \\
\hline Parroy (étang de ) & RA1 & 86 \\
\hline Puylaurent (retenue de ) & RA1 & 91 \\
\hline Charmines-Moux (de ) & RA1 & 95 \\
\hline Champagney (bassin de ) & RA1 & 96 \\
\hline Grand-large (du) & RA1 & 101 \\
\hline Laprade basse (de ) & RA1 & 104 \\
\hline Roujanel & RA1 & 109 \\
\hline Pont du Roi (retenue du ) & RA1 & 110 \\
\hline Saut de vezoles (retenue du ) & RA1 & 112 \\
\hline Torcy Neuf (retenue de ) & RA1 & 113 \\
\hline Torcy Vieux (retenue de ) & RA1 & 114 \\
\hline Settons (barrage des ) & RA1 & 119 \\
\hline Sidiailles (retenue de) & RA1 & 121 \\
\hline Sorme (retenue de la) & RA1 & 124 \\
\hline Vallee (étang de la) & RA1 & 132 \\
\hline Vieilles forges (retenue des ) & RA1 & 133 \\
\hline Vaivre Vesoul (lac du ) & RA1 & 138 \\
\hline Amance (barrage-réservoir aube lac ) & RA2 & 4 \\
\hline Avène (réservoir d') & RA2 & 13 \\
\hline Bimont (lac du ) & RA2 & 18 \\
\hline Caramany (retenue de ) & RA2 & 27 \\
\hline Coiselet (de ) & RA2 & 43 \\
\hline Grangent & RA2 & 57 \\
\hline Laouzas (lac de) & RA2 & 63 \\
\hline Auzon-Temple (barrage-réservoir aube lac ) & RA2 & 64 \\
\hline Madine (étang de la ) & RA2 & 72 \\
\hline Monteynard- Avignonet & RA2 & 76 \\
\hline Notre-Dame de Commiers (de ) & RA2 & 81 \\
\hline
\end{tabular}




\begin{tabular}{|c|c|c|}
\hline Fades Besserve (retenue des ) & RA2 & 100 \\
\hline Saint Cassien (lac de ) & RA2 & 116 \\
\hline Sainte Croix (lac de ) & RA2 & 117 \\
\hline Saint Etienne de Cantalès & RA2 & 118 \\
\hline Saint pardoux & RA2 & 126 \\
\hline Tolla (lac de ) & RA2 & 130 \\
\hline Villerest (retenue de ) & RA2 & 134 \\
\hline Vinça (retenue de ) & RA2 & 135 \\
\hline Vouglans (lac de ) & RA2 & 137 \\
\hline Alesani (retenue de l') & RA3 & 3 \\
\hline Angle guignard (retenue de ) & RA3 & 5 \\
\hline Apremont (retenue d') & RA3 & 7 \\
\hline Arzal (retenue d') & RA3 & 8 \\
\hline Audomarois (marais de l') (Romelaere) & RA3 & 9 \\
\hline Aumée (étang) & RA3 & 11 \\
\hline Beauregard (étang) & RA3 & 17 \\
\hline Bultiere (retenue de la ) & RA3 & 25 \\
\hline Cannes-Ecluse la maserotte (gravière de ) & RA3 & 26 \\
\hline Carcès (lac de ) & RA3 & 28 \\
\hline Cebron (retenue du) & RA3 & 31 \\
\hline Chatillon (étang de ) & RA3 & 36 \\
\hline Chardes (retenue de ) [complexe de Chardes] & RA3 & 41 \\
\hline Codole (retenue de ) & RA3 & 42 \\
\hline Drennec (retenue du) & RA3 & 45 \\
\hline Duc (étang au ) & RA3 & 46 \\
\hline Forge (étang de la) & RA3 & 53 \\
\hline Gouet (retenue du) & RA3 & 56 \\
\hline Graon (retenue du ) & RA3 & 58 \\
\hline Hardouinais (étang de la) & RA3 & 59 \\
\hline Jouarres (étang de ) & RA3 & 61 \\
\hline Jugon (étang de ) [complexe de Ville-Hatte] & RA3 & 62 \\
\hline Jaunay (retenue du) & RA3 & 66 \\
\hline Marillet (lac du ) [complexe du Marillet] & RA3 & 67 \\
\hline Louroux (étang du ) & RA3 & 69 \\
\hline Marcille (étang de ) & RA3 & 73 \\
\hline Miélan (lac de ) & RA3 & 75 \\
\hline Pas du houx (étang du ) & RA3 & 88 \\
\hline Pont-l'Evêque (plan d'eau de ) & RA3 & 90 \\
\hline Rabodanges (barrage de ) & RA3 & 92 \\
\hline Bois Joli (retenue du ) [complexe du Bois Joli] & RA3 & 93 \\
\hline Chapelle Erbrée (retenue de la ) & RA3 & 94 \\
\hline Champsanglard (retenue de ) & RA3 & 97 \\
\hline Valiere (retenue de la ) & RA3 & 98 \\
\hline Réaltor (bassin du ) & RA3 & 99 \\
\hline Rivière Kersan (gravières de la ) & RA3 & 102 \\
\hline Kerne Uhel (retenue de ) & RA3 & 103 \\
\hline Moulin Neuf (retenue du ) & RA3 & 105 \\
\hline Moulin Papon (retenue de ) & RA3 & 106 \\
\hline Moulin ribou (retenue de ) & RA3 & 107 \\
\hline Rochereau & RA3 & 108 \\
\hline Cheze (retenue de la ) & RA3 & 111 \\
\hline
\end{tabular}




\begin{tabular}{|l|c|c|}
\hline Ville-Hatte (retenue de la ) - retenue d'Arguenon & RA3 & 115 \\
\hline Saint Fraimbault de Prieres (retenue de ) & RA3 & $\mathbf{1 2 0}$ \\
\hline Sillonniere (retenue de la ) & RA3 & 122 \\
\hline Saint Michel & RA3 & $\mathbf{1 2 3}$ \\
\hline Sudais (étang de ) & RA3 & 128 \\
\hline Touche Poupard (retenue de la ) & RA3 & $\mathbf{1 3 1}$ \\
\hline Vioreau (étang de ) & RA3 & $\mathbf{1 3 6}$ \\
\hline
\end{tabular}

